\title{
CHARACTERIZATIONS OF THE GENERALIZED CONVEX KERNEL
}

\section{ARTHUR G. SPARKS}

\begin{abstract}
It is well known that the convex kernel $K$ of a set $S$ is the intersection of all maximal convex subsets of $S$. In this paper it is shown that the $n$th order kernel of a compact, simply-connected set $S$ in the plane is an $L_{n}$ set and is, in fact, the intersection of all maximal $L_{n}$ subsets of $S$. Furthermore, it is shown that one does not have to intersect the family of all the maximal $L_{n}$ subsets to obtain the $n$th order kernel, but that any subfamily thereof which covers the set is sufficient.
\end{abstract}

1. Preliminaries. Throughout this paper, all sets will be in $E_{2}$. If $B$ is a set, then $\bar{B}$ will denote its closure, bd $B$ its boundary, and $B^{c}$ its complement. If $x$ and $y$ are points, then $P_{n}(x, y)$ will denote a polygonal $n$-path joining $x$ to $y$.

Let $A$ be a set and let $x$ be in $A$. Then $K(n, x, A)$ will denote the $n$th order kernel of $x$ in $A$. The $n$th order kernel of $A$ will be denoted by $K(n, A)$. For precise definitions of $K(n, x, A)$ and $K(n, A)$, see [2].

Definition. A compact set $S$ is said to be simply-connected if and only if $S^{c}$ is connected.

Hereafter, $S$ will denote a compact, simply-connected set. It is important to remember that if $J$ is a closed Jordan curve in $S$, then the interior of the Jordan curve $J$ is contained in $S$.

Definition. Suppose that $p, q \in S$ and $C(p, q)$ is a polygonal path from $p$ to $q$ in $S$. Then $C(p, q)$ is called a minimal 1-path if $C(p, q)$ is the segment $[p, q]$. Let $k>1$, then $C(p, q)$ is called a minimal $k$-path if $C(p, q)$ is a $k$-path of minimal length joining $p$ to $q$ in $S$ and $p \notin$ $K(k-1, q, S)$.

Several results in a previous paper by this author [2] will be stated for later use.

TheOREM 1.1. Let $A$ be a set and let $B$ be an $L_{n}$ subset of $A$. Then $B$ is contained in a maximal $L_{n}$ subset of $A$.

Theorem 1.2. Suppose $p \in K(m, q, S)$ for some $m$. Then there exists a minimal $k$-path from $p$ to $q$ in $S$ for some $k$ such that $1 \leqq k \leqq m$.

Presented to the Society, August 29, 1969 under the title $A$ characterization of the generalized convex kernel; received by the editors December 7, 1969 and, in revised form, April 15, 1970.

AMS 1970 subject classifications. Primary 52-XX, 52A10.

Key words and phrases. Convex kernel, generalized convex kernel, $L_{n}$ sets. 
Theorem 1.3. Suppose that $p, q \in K(n, x, S)$. Let $C_{k}(p, q)$ be a minimal $k$-path from $p$ to $q$ in $S$, then $C_{k}(p, q) \subset K(n, x, S)$.

THEOREM 1.4. Let $\mathfrak{L}_{n}=\left\{L_{\alpha} \mid \alpha \in \Delta_{n}\right\}$ be the set of all maximal $L_{n}$ subsets of $S$. Then each $L_{\alpha}$ is compact and simply-connected. Furthermore, $\cap \mathfrak{L}_{n}$ is a compact, simply-connected, $L_{n}$ sel.

\section{Characterizations of $K(n, S)$.}

Theorem 2.1. Let $A$ be a compact, $L_{n}$ subset of $S$. Suppose $x \in S$ is such that $A \subset K(n, x, S)$. Then $A \cup\{x\}$ is contained in an $L_{n}$ subset of $S$.

Proof. Let $a \in A$. Since $x \in K(n, a, S)$, it follows from Theorem 1.2 that there exists a minimal $k(a)$-path $C_{k(a)}(a, x)$ joining $a$ to $x$ in $S$, where $k(a) \leqq n$. Let $G=\bigcup\left\{C_{k(a)}(a, x) \mid a \in A\right\}$ and let $D$ be the smallest compact, simply-connected set in $S$ which contains $G$.

Suppose that $p, q \in G$. Then there exist $a_{1}, a_{2} \in A$ such that $p \in$ $C_{k\left(a_{1}\right)}\left(a_{1}, x\right)$ and $q \in C_{k\left(a_{2}\right)}\left(a_{2}, x\right)$. It is clear that $x \in K\left(n, a_{1}, D\right)$ and $a_{2} \in$ $K\left(n, a_{1}, D\right)$. Since $C_{k\left(a_{2}\right)}\left(a_{2}, x\right) \subset D$, it follows from Theorem 1.3 that $C_{k\left(a_{2}\right)}\left(a_{2}, x\right) \subset K\left(n, a_{1}, D\right)$. In particular, $q \in K\left(n, a_{1}, D\right)$ and thus $a_{1} \in K(n, q, D)$. Now since $x \in K(n, q, D)$ and $C_{k\left(a_{1}\right)}\left(a_{1}, x\right)$ is also a minimal $k\left(a_{1}\right)$-path in $D$, it follows again by Theorem 1.3 that $C_{k\left(a_{1}\right)}\left(a_{1}, x\right) \subset K(n, q, D)$. In particular, it is true that $p \in K(n, q, D)$.

Now suppose that $p, q \in$ bd $D$. It is clear that bd $D \subset \bar{G}$. Since $p, q \in \bar{G}$, there exist sequences $\left\{p_{i}\right\}$ and $\left\{q_{i}\right\}$ in $G$ such that $\left\{p_{i}\right\} \rightarrow p$ and $\left\{q_{i}\right\} \rightarrow q$.

Let $i$ and $j$ be arbitrary, then from the preceding it follows that $p_{i} \in K\left(n, q_{j}, D\right)$ since $p_{i}, q_{j} \in G$. Now $i$ arbitrary implies that $\left\{p_{i}\right\}$ $\subset K\left(n, q_{j}, D\right)$. It has been shown by Bruckner and Bruckner [1], that $K\left(n, q_{j}, D\right)$ is compact. Thus, it follows that $p \in K\left(n, q_{j}, D\right)$. Hence, $q_{j} \in K(n, p, D)$ where $j$ is arbitrary and thus $\left\{q_{i}\right\} \subset K(n, p, D)$. As before, $K(n, p, D)$ is compact and hence $q \in K(n, p, D)$. Since $p, q \in$ bd $D$ were arbitrary, it follows by another result of Bruckner and Bruckner [1] that $D$ is an $L_{n}$ set. It is clear that $A \cup\{x\} \subset D \subset S$. This completes the proof.

THEOREM 2.2. Let $\mathscr{L}_{n}=\left\{L_{\alpha} \mid \alpha \in \Delta_{n}\right\}$ be the set of all maximal $L_{n}$ subsets of $S$. Then $K(n, S)=\bigcap_{n}$.

Proof. Clearly, $\cap \mathfrak{L}_{n} \subset K(n, S)$.

Suppose that $x$ is in $K(n, S)$ but not in $L_{\alpha}$, for some $\alpha \in \Delta_{n}$. Since $L_{\alpha} \subset K(n, x, S)$, Theorem 2.1 implies that $L_{\alpha}$ and $x$ are both contained in an $L_{n}$ subset of $S$, contradicting the maximality of $L_{\alpha}$. Hence, $K(n, S) \subset \cap \mathfrak{L}_{n}$. 
Combining the above, the desired result is obtained.

The same technique can be used to prove the following result:

THEOREM 2.3. Let $\mathscr{L}_{n}^{\prime} \subset \mathfrak{L}_{n}$ be such that $\bigcup_{\mathfrak{L}_{n}^{\prime}}=S$, then $\cap \mathfrak{L}_{n}^{\prime}=K(n, S)$.

THEOREM 2.4. The set $K(n, S)$ is an $L_{n}$ set.

Proof. Combine Theorems 1.4 and 2.2.

\section{REFERENCES}

1. A. M. Bruckner and J. B. Bruckner, Generalized convex kernels, Israel J. Math. 2 (1964), 27-32. MR 30 \#1448.

2. A. G. Sparks, Intersections of maximal $L_{n}$ sets, Proc. Amer. Math. Soc. 24 (1970), 245-250.

3. F. A. Toranzos, Radial functions of convex and star-shaped bodies, Amer. Math. Monthly 74 (1967), 278-280. MR 34 \#8279.

Clemson University, Clemson, South Carolina 29631

Georgia Southern College, Statesboro, Georgia 30458 